\title{
Hedging and Portfolio Optimization in Financial Markets with a Large Trader
}

\author{
By Peter BanK ${ }^{\dagger}$ And Dietmar Baum ${ }^{\ddagger}$
}

\begin{abstract}
We introduce a general continuous-time model for an illiquid financial market where the trades of a single large investor can move market prices. The model is specified in terms of parameter dependent semimartingales, and its mathematical analysis relies on the non-linear integration theory of such semimartingale families. The Itô-Wentzell formula is used to prove absence of arbitrage for the large investor, and using approximation results for stochastic integrals, we characterize the set of approximately attainable claims. We furthermore show how to compute superreplication prices and discuss the large investor's utility maximization problem.
\end{abstract}

AMS 2000 subject classification. 60H05, 60H30, 91B28, $91 \mathrm{~B} 70$

Key words and phrases. large investor, feedback effect, Itô-Wentzell formula, parameter dependent semimartingales, uniform approximation of stochastic integrals

Acknowledgement. We would like to thank Hans Föllmer, Rüdiger Frey, Peter Imkeller, and Martin Schweizer for helpful discussions and comments. We also would like to acknowledge the helpful suggestions of an anonymous referee.

$\dagger$ Humboldt University of Berlin. Support of Deutsche Forschungsgemeinschaft through SFB 373, "Quantification and Simulation of Economic Processes", is gratefully acknowledged.

$\ddagger$ Bank of America, London. 


\section{Introduction}

Most financial market models considered in Mathematical Finance assume perfect elasticity for the supply and demand of traded assets so that orders of arbitrary size do not affect asset prices. This assumption is justified as long as one considers 'small' investors whose trading volume is easily covered by market liquidity. However, if there is a 'large' investor in the market, whose orders involve a significant part of the available shares, market prices will no longer evolve independently of the trading strategies chosen by this 'big player'. It then becomes an issue how the large investor should account for this feedback effect when he chooses his portfolio strategy.

A number of suggestions on how to formalize this problem in a mathematical model have been made. Jarrow $(1992,1994)$ proposes a discrete-time framework where prices depend on the large trader's activities via a reaction function of his instantaneous holdings. Frey and Stremme (1997) develop a continuous time analogue of this framework which essentially forms also the basis of Platen and Schweizer (1998) and Papanicolaou and Sircar (1998). Kyle (1985) and Back (1992) use an equilibrium approach to obtain similar asset price dynamics in the presence of an insider. In contrast to these contributions in which prices directly depend on the large investor's holdings via a reaction function, Cuoco and Cvitanic (1998) and Cvitanic and Ma (1996) study a diffusion model for the price dynamics where feedback is rather indirect as only the drift and volatility coefficients depend on the large investor's trading strategy. In the present paper, we assume that there is a family of semimartingales $P^{\vartheta}(\vartheta \in \mathbb{R})$ which specify the dynamics of the illiquid asset when the large investor's position is kept constant at a certain level $\vartheta$. This yields a time-varying reaction function $\vartheta \mapsto P^{\vartheta}$ which allows us to define the asset price dynamics in the same manner as in the reaction diffusion setting of Frey and Stremme (1997). This model is similar to an approach developed by Çetin, Jarrow, and Protter (2002) and Çetin, Jarrow, Protter, and Warachka (2002). A conceptually and methodologically crucial difference between their approach and ours, however, is that in their model the price effect of an order is limited to the very moment when the order is placed in the market, while in our reaction function model the effect of an order will persist until the next order makes asset prices follow a possibly different dynamics.

The feedback between asset prices and the large investor's trading strategy entails two competing aspects. On the one hand, the large investor might be able to (ab)use his market power in order to manipulate market prices in his favor. On the other hand, illiquidity causes transaction costs since the large investor's orders are only exercised 
after prices have adversely adjusted to them so that the large investor always has to trade on the 'bad' side. It is therefore interesting to investigate if there are any arbitrage opportunities for the large investor. In a mathematically rigorous manner, this question was first addressed in the discrete-time account of Jarrow (1992) who proved absence of arbitrage for the large investor under the assumption of absence of arbitrage for small investors in periods where the large investor does not trade. Using an approximation argument, this result was subsequently extended by Bierbaum (1997) to (essentially) the continuous-time reaction function setting of Frey and Stremme (1997). Instead of building on Jarrow's discrete-time result, we give a more direct martingale theoretic proof for absence of arbitrage under essentially the same condition. Our approach is based on the Itô-Wentzell formula for parameter dependent semimartingales which allows us to give an explicit decomposition of the real wealth dynamics into the profits and losses which are due to exogenous random shocks and into transaction costs caused by illiquidity.

The real wealth dynamics reveals that in order to avoid transaction costs, the large investor should use continuous trading strategies of bounded variation. Thus, it is interesting to determine which payoff profiles can be approximately attained by such strategies. We answer this question by showing how to uniformly approximate an arbitrary stochastic integral by other stochastic integrals with continuous integrands of bounded variation. This supplements an approximation result for stochastic integrals obtained in Levental and Skorohod (1997). The economic implication of this result is that our large investor model inherits many properties of its underlying primal small investor models. Indeed, it turns out that under some natural assumptions attainable claims in a suitable small investor model become approximately attainable in our large investor setting. Similarly, one can reduce the computation of superreplication prices from the large investor's view point to the computation of superreplication prices in a small investor model. As a consequence, we obtain that the large investor can obtain the same utility as a small investor in an associated model.

An outline of the present paper is as follows. Section 1 introduces a general semimartingale model for the price fluctuations of an illiquid financial asset. Section 2 provides the dynamics of the real wealth process and proves absence of arbitrage for the large investor. Section 3 contains our approximation result for stochastic integrals and characterizes approximately attainable claims; it furthermore discusses the large investor's utility maximization problem. Section 4 shows how to compute superreplication prices for manipulable derivatives. Section 5 concludes. Some more technical arguments are relegated to the appendix. 


\section{Price dynamics in the presence of a large investor}

We consider a financial market containing a risky stock and a riskless bond paying interest at some rate $r$. There is one large investor in the market whose trades may affect the price process of the risky asset, but not the riskless interest rate. Hence, by passing to discounted prices, we may assume without loss of generality that the interest rate is fixed at $r \equiv 0$. To specify our model for the price evolution of the imperfectly elastic risky asset, let us fix a filtered probability space $(\Omega, \mathscr{F}, \mathbb{P}, \mathbb{F})$ with a filtration $\mathbb{F}=\left(\mathscr{F}_{t}\right)_{0 \leq t \leq T}$ satisfying the usual conditions of right continuity and completeness; $\mathscr{F}_{0}$ is trivial modulo $\mathbb{P}$. As a primitive for our model, we assume to be given a family of continuous semimartingales $P^{\vartheta}=\left(P_{t}^{\vartheta}\right)_{0 \leq t \leq T}(\vartheta \in \mathbb{R})$. The process $P^{\vartheta}$ is interpreted as a model for the price fluctuations of the risky asset given that the large investor holds a constant stake of $\vartheta$ shares in this asset. If the large investor chooses a time-varying strategy $\theta=\left(\theta_{t}\right)_{0 \leq t \leq T}$, the resulting asset price evolution can then be modelled as

$$
P_{t}^{\theta}=P\left(\theta_{t}, t\right) \triangleq P_{t}^{\theta_{t}} \quad(0 \leq t \leq T) .
$$

Examples 1.1 Let us give two important examples which are covered by this setting:

(i) A simple example is a classical Black-Scholes model with a Brownian motion $B$ and parameter dependent drift and volatility:

$$
d P_{t}^{\vartheta}=P_{t}^{\vartheta}\left(\mu_{t}^{\vartheta} d t+\sigma_{t}^{\vartheta} d B_{t}\right) \quad(\vartheta \in \mathbb{R}) .
$$

Note that even though this dynamics very much resembles the setting of Cvitanic and Ma (1996) and Cuoco and Cvitanic (1998) there is a crucial difference between our model and their diffusion model: whereas in the latter model only the drift and volatility parameter will change immediately when the large investor changes his position, in our model also the asset price itself will change in general. For instance, when the large investor liquidates a position of $\theta_{T}$ shares, at time $T$ say, this does not affect the asset price immediately at time $T$ in the model of Cvitanic et al.; in contrast, in our model the price will immediately move from $P_{T}^{\theta_{T}}$ before liquidation to $P_{T}^{0}$ after liquidation.

(ii) Also the reaction diffusion setting of, e.g., Frey and Stremme (1997) is included in this setting since in such a framework we may choose

$$
P_{t}^{\vartheta}=\psi\left(t, B_{t}, \vartheta\right) \quad(\vartheta \in \mathbb{R})
$$

for some smooth function $\psi:[0, T] \times \mathbb{R} \times \mathbb{R} \rightarrow \mathbb{R}$. 
Plainly, without additional assumptions, asset price fluctuations driven by (1) may be unreasonably strong unless we restrict the class of strategies $\theta$ at the large investors disposal. A convenient restriction is that the large investor is confined to use strategies which are semimartingales with respect to $(\mathbb{P}, \mathbb{F})$. Indeed, in conjunction with a smoothness assumption on $\vartheta \mapsto P^{\vartheta}$ this will ensure that, as usual, asset prices follow a general semimartingale dynamics; see Corollary 1.5 .

Definition 1.2 A family of semimartingales $S^{\vartheta}(\vartheta \in \mathbb{R})$ is called smooth if it satisfies the following conditions:

(i) Every $S^{\vartheta}(\vartheta \in \mathbb{R})$ is a continuous semimartingale with Doob-Meyer decomposition $S^{\vartheta}=M^{\vartheta}+A^{\vartheta}$.

(ii) The covariation processes $\left[M^{\vartheta}, M^{\vartheta^{\prime}}\right]\left(\vartheta, \vartheta^{\prime} \in \mathbb{R}\right)$ and compensators $A^{\vartheta}(\vartheta \in \mathbb{R})$ can be chosen so that for every $(\omega, t) \in \Omega \times[0, T]$ we have

- $\left[M^{\vartheta}, M^{\vartheta^{\prime}}\right]_{t}(\omega)$ is twice continuously differentiable in $\left(\vartheta, \vartheta^{\prime}\right)$, and the respective second derivatives are locally Hölder-continuous for some index $\delta>0$,

- $A_{t}^{\vartheta}(\omega)$ is once continuously differentiable in $\vartheta \in \mathbb{R}$.

(iii) There exists an adapted process $A$ with increasing and continuous paths such that each covariation process $\left[M^{\vartheta}, M^{\vartheta^{\prime}}\right]\left(\vartheta, \vartheta^{\prime} \in \mathbb{R}\right)$ and every compensator $A^{\vartheta}(\vartheta \in \mathbb{R})$ is absolutely continuous with respect to $A$.

As shown in Kunita (1990), Chapter 3.1, we can modify the martingales occurring in the Doob-Meyer decompositions $S^{\vartheta}=M^{\vartheta}+A^{\vartheta}(\vartheta \in \mathbb{R})$ associated with a smooth semimartingale family $S$ so that $M_{t}^{\vartheta}(\omega)$ becomes twice continuously differentiable in $\vartheta \in \mathbb{R}$ for any $(\omega, t) \in \Omega \times[0, T]$. Thus, whenever in the sequel we consider a smooth family of semimartingales we may and will work with such a nice version.

Proposition 1.3 (Itô-Wentzell formula) Let $S^{\vartheta}(\vartheta \in \mathbb{R})$ be a smooth family of semimartingales. Then, for any RCLL semimartingale $\theta$, the process $S\left(\theta_{t}, t\right) \triangleq S_{t}^{\theta_{t}}(0 \leq$ $t \leq T)$ is again a semimartingale and its dynamics is described by

$$
\begin{aligned}
& S\left(\theta_{t}, t\right)-S\left(\theta_{0-}, 0\right)= \\
& \quad \int_{0}^{t} S\left(\theta_{s-}, d s\right)+\int_{0}^{t} S^{\prime}\left(\theta_{s-}, s\right) d \theta_{s}+\left[\int_{0}^{\cdot} S^{\prime}\left(\theta_{s-}, d s\right), \theta\right]_{t} \\
& +\frac{1}{2} \int_{0}^{t} S^{\prime \prime}\left(\theta_{s-}, s\right) d[\theta]_{s}^{c}+\sum_{0 \leq s \leq t}\left\{\Delta S\left(\theta_{s}, s\right)-S^{\prime}\left(\theta_{s-}, s\right) \Delta \theta_{s}\right\}
\end{aligned}
$$


where $\int_{0} S\left(\theta_{s-}, d s\right)$ denotes the stochastic integral of $\theta_{-}$with respect to the semimartingale kernel $S(\vartheta, d s)$ and where all derivatives are taken with respect to $\vartheta$.

Proof : For continuous $\theta$ this follows from Theorem 3.3.1 in Kunita (1990). For $\theta$ with jumps the proof is a straightforward extension of the argument in Kunita (1990), e.g., using the techniques of Protter (1990) to prove the general Itô-formula with jumps.

Remark 1.4 Kunita (1990), Chapter 3.2, gives a detailed account of how to construct stochastic integrals with respect to a semimartingale kernel $S(\vartheta, d s)$ by starting from the elementary definition

$$
\int_{0}^{t} S\left(\theta_{s}, d s\right) \triangleq \sum_{i}\left\{S\left(\theta_{s_{i} \wedge t}, s_{i+1} \wedge t\right)-S\left(\theta_{s_{i} \wedge t}, s_{i} \wedge t\right)\right\} \quad(0 \leq t \leq T)
$$

for simple integrands of the form $\theta=\sum_{i} \theta_{i} 1_{\left(s_{i}, s_{i+1}\right]}$ with $0 \leq s_{0}<\ldots \leq s_{n} \leq T$ and $\theta_{i} \in L^{0}\left(\mathscr{F}_{s_{i}}\right)$. The quadratic variation of $\int_{0} S\left(\theta_{s}, d s\right)$ is given by

$$
\left[\int_{0} S\left(\theta_{s}, d s\right)\right]_{t}=\int_{0}^{t} a\left(\theta_{s}, \theta_{s}, s\right) d A_{s} \quad(0 \leq t \leq T)
$$

where $A$ is the dominating increasing process of Definition 1.2 (iii) and $a=a\left(\vartheta, \vartheta^{\prime}, \omega, s\right.$ ) denotes a $\left(\vartheta, \vartheta^{\prime}\right)$-continuous choice of the densities $d\left[M^{\vartheta}, M^{\vartheta^{\prime}}\right]_{s} / d A_{s}\left(\vartheta, \vartheta^{\prime} \in \mathbb{R}\right)$.

An immediate consequence of the preceding proposition is

Corollary 1.5 If the primal semimartingales $P^{\vartheta}(\vartheta \in \mathbb{R})$ define a smooth semimartingale family, then the asset price process (1) follows a semimartingale dynamics for any semimartingale strategy $\theta$ of the large investor.

To ensure reasonable price fluctuations, we therefore introduce the following standing assumption:

Assumption 1 The family of semimartingales $P^{\vartheta}(\vartheta \in \mathbb{R})$ is smooth in the sense of Definition 1.2.

\section{Wealth dynamics and no arbitrage}

A crucial viability question in every financial market model is whether it allows for profits without risk, i.e., for arbitrage. For models with small investors this question 
has been solved in great generality by the celebrated fundamental theorem of asset pricing; see Delbaen and Schachermayer (1998) and the references therein. Rather few investigations, however, deal with this question from the perspective of a large investor whose orders directly influence stock prices. In a discrete time framework, Jarrow (1992) proves absence of arbitrage for large investors under the assumption that small investors cannot produce riskless profits in periods where the large investor does not trade. Using an approximation argument, Bierbaum (1997) extends this result (essentially) to the reaction diffusion model of Frey and Stremme (1997).

In order to investigate the question of existence of arbitrage opportunities in our more general model, we first want to clarify the wealth dynamics that are generated by selffinancing portfolio strategies. To this end, consider a semimartingale strategy $\theta=\left(\theta_{t}\right)_{0 \leq t \leq T}$ for the large investor which describes the number of shares held by the investor at each point in time. His (discounted) holdings in the bank account $\beta^{\theta}$ will then evolve according to the dynamics

$$
\beta_{t}^{\theta}=\beta_{0-}-\int_{0}^{t} P\left(\theta_{s-}, s\right) d \theta_{s}-[P(\theta, .), \theta]_{t} \quad(0 \leq t \leq T) .
$$

Here, the quadratic variation term implies that asset prices are affected by the large investor's orders before these are actually exercised. Indeed, assume, for instance, the order is of size $\Delta \theta_{t}>0$. Then the large investor's bank account will be charged

$$
\Delta \beta_{t}^{\theta}=-P\left(\theta_{t-}, t\right) \Delta \theta_{t}-\Delta P\left(\theta_{t}, t\right) \Delta \theta_{t}=-P\left(\theta_{t}, t\right) \Delta \theta_{t}
$$

and so he has to pay $P\left(\theta_{t}, t\right)$ for each of his $\Delta \theta_{t}$ ordered shares. This price will be no less than the pre-order price $P\left(\theta_{t-}, t\right)$ if we impose the following natural condition.

Assumption 2 Asset prices are non-decreasing with respect to the large investor's position: $P^{\vartheta} \leq P^{\vartheta^{\prime}}$ for $\vartheta \leq \vartheta^{\prime}$.

This assumption is crucial for our model to exclude trivial arbitrage opportunities. In fact, suppose that at some point in time $t$ we have $P(\vartheta, t)>P\left(\vartheta^{\prime}, t\right)$ for some $\vartheta<\vartheta^{\prime}$. Then a large investor could increase his number of shares from $\vartheta$ to $\vartheta^{\prime}$ at costs $P\left(\vartheta^{\prime}, t\right)\left\{\vartheta^{\prime}-\vartheta\right\}$. Having done so, he very shortly afterwards could reduce his number of shares to the original amount $\vartheta$ receiving about $P(\vartheta, t)\left\{\vartheta^{\prime}-\vartheta\right\}$. The overall proceeds from such an in-and-out strategy would be $\left\{P(\vartheta, t)-P\left(\vartheta^{\prime}, t\right)\right\}\left\{\vartheta^{\prime}-\vartheta\right\}>0$, i.e., the large investor could make a profit without taking risk.

Let us now describe the wealth dynamics induced by a large investor strategy. Assume that at some time $t$ the large investor has $\beta_{t}^{\theta}$ in the bank account and a stock 
position of $\theta_{t}$ shares. The book or paper value of his portfolio is then

$$
W_{t}^{\theta} \triangleq \beta_{t}^{\theta}+P\left(\theta_{t}, t\right) \theta_{t}
$$

However, if the investor was forced to liquidate his stock position immediately by a single block trade, he would not be able to trade his shares at price $P\left(\theta_{t}, t\right)$ but only at $P(0, t)$ and, thus, the block liquidation value of his position would be

$$
\tilde{V}_{t}^{\theta} \triangleq \beta_{t}^{\theta}+P(0, t) \theta_{t}
$$

Clearly, selling a huge position of shares en bloc can be very disadvantageous as prices will fall before such large sell orders are exercised. Hence, the difference between book value and block liquidation value $W_{t}^{\theta}-\tilde{V}_{t}^{\theta}=\left(P\left(\theta_{t}, t\right)-P(0,1)\right) \theta_{t} \geq 0$ can be quite substantial. In such a situation, it would be more convenient to split the order into smaller packages which are then sold one after the other over a small time period. In our model, it is not difficult to see that in the limit as the packages become ever smaller and as the duration for liquidation tends to 0 the proceeds from such a fast liquidation strategy become

$$
L(\vartheta, t) \triangleq \int_{0}^{\vartheta} P(x, t) d x
$$

where $\vartheta=\theta_{t}$ denotes the large investor's number of shares before liquidation.

Remark 2.1 The above asymptotic description of liquidation proceeds also occurs in Back (1992) and Schönbucher and Wilmott (2000). Note that here and in the sequel we follow the usual sign-convention $\int_{a}^{b} \ldots \triangleq-\int_{b}^{a} \ldots$ in case $b<a$.

Note that under Assumption 2 the asymptotic liquidation proceeds $L(\vartheta, t)$ from a position of $\vartheta$ shares always lies in between the position's book value $P(\vartheta, t) \vartheta$ and its block liquidation value $P(0, t) \vartheta$. This leads us to define the asymptotically realizable or real wealth achieved by a trading strategy $\theta$ until time $t$ as

$$
V_{t}^{\theta} \triangleq \beta_{t}^{\theta}+L\left(\theta_{t}, t\right)
$$

Plainly, book value, block liquidation value and real wealth coincide whenever $\theta_{t}=0$.

It turns out that in contrast to the dynamics of the book value, the dynamics of the realizable wealth is quite tractable and transparent. The explicit dynamics we obtain for the realizable wealth in the following lemma will be used extensively in the remainder of the text when dealing with the problems of hedging and portfolio optimization from the perspective of a large investor. 
Lemma 2.2 For any selffinancing semimartingale strategy $\theta$, the dynamics of the real wealth process $V^{\theta}$ is given by

$$
\begin{aligned}
V_{t}^{\theta}-V_{0-}^{\theta}= & \int_{0}^{t} L\left(\theta_{s-}, d s\right)-\frac{1}{2} \int_{0}^{t} P^{\prime}\left(\theta_{s-}, s\right) d[\theta]_{s}^{c} \\
& -\sum_{0 \leq s \leq t} \int_{\theta_{s-}}^{\theta_{s}}\left\{P\left(\theta_{s}, s\right)-P(x, s)\right\} d x .
\end{aligned}
$$

Proof : By assumption on $P$, the family of semimartingales $L^{\vartheta}(\vartheta \in \mathbb{R})$ defined by (4) is smooth. Hence, the Itô-Wentzell formula of Proposition 1.3 yields that $L\left(\theta_{t}, t\right)$ is a semimartingale with dynamics

$$
\begin{aligned}
L\left(\theta_{t}, t\right)= & L\left(\theta_{0-}, 0\right)+\int_{0}^{t} L\left(\theta_{s-}, d s\right)+\int_{0}^{t} P\left(\theta_{s-}, s\right) d \theta_{s} \\
& +\left[\int_{0} P\left(\theta_{s-}, d s\right), \theta\right]_{t}+\frac{1}{2} \int_{0}^{t} P^{\prime}\left(\theta_{s-}, s\right) d[\theta]_{s}^{c} \\
& +\sum_{0 \leq s \leq t}\left\{\int_{\theta_{s-}}^{\theta_{s}} P(x, s) d s-P\left(\theta_{s-}, s\right) \Delta \theta_{s}\right\} .
\end{aligned}
$$

An application of the Itô-Wentzell formula to $P^{\theta}=\left(P\left(\theta_{t}, t\right)\right)_{0 \leq t \leq T}$ allows us to compute $\left[P^{\theta}, \theta\right]$, and we find

$$
\begin{aligned}
\beta_{t}^{\theta}= & \beta_{0-}-\int_{0}^{t} P\left(\theta_{t-}, t\right) d \theta_{t}-\left[\int_{0}^{\cdot} P\left(\theta_{s-}, d s\right), \theta\right]_{t} \\
& -\int_{0}^{t} P^{\prime}\left(\theta_{s-}, s\right) d[\theta]_{s}^{c}-\sum_{0 \leq s \leq t} \Delta P\left(\theta_{s}, s\right) \Delta \theta_{s} .
\end{aligned}
$$

When adding the preceding two equations, several terms cancel out and we obtain the claimed formula for $V_{t}^{\theta}=\beta_{t}^{\theta}+L\left(\theta_{t}, t\right)$.

As shown by the preceding lemma, the real wealth dynamics $V^{\theta}$ can be decomposed into three parts. The first part, $\int_{0}^{t} L\left(\theta_{s-}, d s\right)$, accounts for profits or losses from stock price fluctuations which are due to exogenous random shocks. The second and third part can be viewed as transaction costs due to limited liquidity. Indeed, by Assumption 2 , the large investor always has to trade on the 'bad' side since his orders always adversely affect the stock price before being exercised. The induced transaction costs become particularly obvious in the case of block orders whose transaction costs are reflected in the jump term $\sum_{0 \leq s \leq t} \int_{\theta_{s-}}^{\theta_{s}}\left\{P\left(\theta_{s}, s\right)-P(x, s)\right\} d x \geq 0$. Additionally, it turns out that trading in a fluctuating manner also produces transaction costs; these costs are described by the quadratic variation term $\frac{1}{2} \int_{0}^{t} P^{\prime}\left(\theta_{s-}, s\right) d[\theta]_{s}^{c} \geq 0$. Finally, it is interesting to 
see that 'tame' trading strategies whose trajectories $\left(\theta_{t}\right)_{0 \leq t \leq T}$ are continuous and of bounded variation do not produce transaction costs in this sense - an observation which is important in Back's (1992) analysis of insider trading and which will also be crucial in the sequel when we are going to discuss the large investor's hedging problem.

As a consequence, the large investor can deliberately move market prices only at certain costs, and it becomes an interesting question under which conditions these costs actually suffice to rule out any market manipulation strategy which would allow the large investor to produce riskless profits, i.e., to have an arbitrage opportunity due to his market power. In order to discuss this issue properly, we first have to exclude any doubling strategies from our considerations by introducing a suitable notion of admissible strategies — just like in the standard small investor framework. For our framework, the real wealth dynamics (5) suggests to call a selffinancing semimartingale strategy $\theta$ admissible if the induced profits or losses from exogenous shocks $\int_{0}^{\cdot} L\left(\theta_{s-}, d s\right)$ are bounded from below by some real constant. Indeed, for the special case where the large investor is in fact an 'ordinary' small investor in the sense that $P_{t}^{\vartheta}$ does not depend on $\vartheta$, this reduces to the usual notion of admissibility.

In the standard small investor setting, absence of arbitrage is (essentially) equivalent to the existence of an equivalent local martingale measure. For our purposes, the following assumption will be convenient.

Assumption 3 There exists a measure $\mathbb{P}^{*} \approx \mathbb{P}$ which simultaneously is a local martingale measure for all our primal processes $P^{\vartheta}(\vartheta \in \mathbb{R})$.

By the fundamental theorem of asset pricing, this assumption implies that small investors cannot make any riskless profits in periods where the large investor does not trade. More precisely, the fundamental theorem shows that no arbitrage for small investors in this sense is essentially equivalent to the existence of an equivalent local martingale measure $\mathbb{P}^{\vartheta}$ for any primal process $P^{\vartheta}(\vartheta \in \mathbb{R})$. Thus, Assumption 3 is slightly stronger than the assumption of no arbitrage for small investors since it implies that we can choose $\mathbb{P}^{\vartheta}=\mathbb{P}^{*}$ independently of $\vartheta \in \mathbb{R}$. In our diffusion example 1.1(i), this corresponds to the condition that the market price of risk $\left(\mu_{t}^{\vartheta}-r\right) / \sigma_{t}^{\vartheta}$ associated with the exogenous risk factor $d B_{t}$ does not depend on the large investor's position $\vartheta$.

Interestingly, in conjunction with our previous monotonicity assumption, Assumption 3 also suffices to rule out riskless profits for the large investor.

Theorem 2.3 Under Assumptions 1 3, there exists no arbitrage opportunity for the large investor in the class of admissible strategies, i.e., there is no admissible strategy $\theta$ 
such that

$$
\mathbb{P}\left[V_{T}^{\theta} \geq V_{0-}^{\theta}\right]=1 \quad \text { and } \quad \mathbb{P}\left[V_{T}^{\theta}>V_{0-}^{\theta}\right]>0 .
$$

Proof : As $P(\vartheta, t)$ is increasing in $\vartheta$ by assumption, the transaction cost terms in our real wealth dynamics are non-negative. Hence, for any selffinancing large investor strategy $\theta$, the real wealth process $V^{\theta}$ is bounded from above by $V_{0-}^{\theta}+\int_{0}^{\cdot} L\left(\theta_{s-}, d s\right)$. The preceding stochastic integral is a local martingale under $\mathbb{P}^{*}$, and, provided $\theta$ is admissible, it is also bounded from below and thus a $\mathbb{P}^{*}$-supermartingale. It follows that, for admissible $\theta$, we have $\mathbb{E}^{*} V_{T}^{\theta} \leq V_{0-}^{\theta}$. As $\mathbb{P}^{*} \approx \mathbb{P}$, this proves that $\theta$ cannot be an arbitrage opportunity for the large investor.

\section{Approximation of stochastic integrals and approx- imate attainability}

An interesting consequence of the real wealth dynamics (5) is the observation that continuous strategies of bounded variation do not incur transaction costs for the large investor. It is therefore important to determine which final payoffs the investor can attain by following such 'tame' strategies. To answer this question, it is useful to introduce the following concepts:

Definition 3.1 A contingent claim $H \in L^{0}\left(\mathscr{F}_{T}\right)$ is called attainable modulo transaction costs for initial capital $v$ if $H=v+\int_{0}^{T} L\left(\theta_{s}, d s\right)$ almost surely for some $L$-integrable predictable process $\theta$ for which $\int_{0}^{\cdot} L\left(\theta_{s}, d s\right)$ is uniformly bounded from below.

A contingent claim $H \in L^{0}\left(\mathscr{F}_{T}\right)$ is called approximately attainable for initial capital $v$ if for any $\varepsilon>0$ there exists an admissible large investor strategy $\theta^{\varepsilon}$ such that $V^{\theta^{\varepsilon}}$ with $V_{0-}^{\theta^{\varepsilon}}=v$ satisfies

$$
\left|H-V_{T}^{\theta^{\varepsilon}}\right| \leq \varepsilon \quad \mathbb{P}-\text { a.s. } .
$$

Thus, any claim which is approximately attainable for some initial capital can be superreplicated in the usual sense when starting with only a little more capital.

Remark 3.2 The preceding definition only refers to contingent claims $H$ whose payoff is completely determined by the exogenous risk factors and which can thus be represented by a real-valued contingent claim $H \in L^{0}\left(\mathscr{F}_{T}\right)$. Many real claims, though, explicitly depend on the evolution of the underlying asset, and their payoff can therefore be directly affected by the large investor. These more general claims will be discussed in Section 4 below. 
With this terminology we can state the main result of this section as follows.

Theorem 3.3 Under Assumption 1, any contingent claim $H \in L^{0}\left(\mathscr{F}_{T}\right)$ which is attainable modulo transaction costs is approximately attainable with the same initial capital.

The PROOF of this theorem follows immediately from the following strong approximation result for stochastic integrals which may be of independent mathematical interest.

Theorem 3.4 Assume $L^{\vartheta}(\vartheta \in \mathbb{R})$ is a smooth family of semimartingales. Let $\theta$ be an $L$-integrable, predictable process and fix $\vartheta_{0} \in L^{0}\left(\mathscr{F}_{0}\right), \vartheta_{T} \in L^{0}\left(\mathscr{F}_{T-}\right)$. Then, for any $\varepsilon>0$, there exists a predictable process $\theta^{\varepsilon}$ with continuous paths of bounded variation such that $\theta_{0}^{\varepsilon}=\vartheta_{0}, \theta_{T}^{\varepsilon}=\vartheta_{T}$ and

$$
\sup _{0 \leq t \leq T}\left|\int_{0}^{t} L\left(\theta_{s}, d s\right)-\int_{0}^{t} L\left(\theta_{s}^{\varepsilon}, d s\right)\right| \leq \varepsilon \quad \mathbb{P}-\text { a.s. }
$$

Remark 3.5 The preceding theorem can be viewed as a supplement to a result in Levental and Skorohod (1997). These authors construct piecewise constant RCLL-strategies which uniformly approximate a given stochastic integral in the special case where the integrator is given by a continuous semimartingale $L$, i.e., $L(\vartheta, d s)=\vartheta d L_{s}$.

We will prove Theorem 3.4 by a Borel-Cantelli argument which is based on the following lemma whose proof is given in the appendix.

Lemma 3.6 Let $\theta$ be as in Theorem 3.4, fix a stopping time $\tau \leq T$ and consider a random variable $\vartheta_{\tau} \in L^{0}\left(\mathscr{F}_{\tau}\right)$ with $\vartheta_{\tau}=\vartheta_{T}$ on $\{\tau=T\}$. For any $\varepsilon>0$, there exists a predictable process $\theta^{\varepsilon, \tau, \vartheta_{\tau}}$ whose paths are continuous and of bounded variation over $[\tau, T]$ such that $\theta_{\tau}^{\varepsilon, \tau, \vartheta_{\tau}}=\vartheta_{\tau}, \theta_{T}^{\varepsilon, \tau, \vartheta_{\tau}}=\vartheta_{T}$ and

$$
\mathbb{P}\left[\sup _{\tau \leq t \leq T}\left|\int_{\tau}^{t} L\left(\theta_{s}, d s\right)-\int_{\tau}^{t} L\left(\theta_{s}^{\varepsilon, \tau, \vartheta_{\tau}}, d s\right)\right| \geq \varepsilon\right] \leq \varepsilon .
$$

Let us now give the

Proof of Theorem 3.4 Let $\varepsilon_{n} \triangleq \varepsilon / 2^{n}(n=0,1, \ldots)$, put $\tau_{0} \triangleq 0$ and define $\theta_{0}^{\varepsilon} \triangleq \vartheta_{0}$. We are going to extend the definition of $\theta^{\varepsilon}$ inductively. So assume that for some $n \in$ $\{0,1, \ldots\}$ we already have constructed $\theta^{\varepsilon}$ on the random interval $\left[0, \tau_{n}\right]$ where $\tau_{n}$ is a suitably constructed stopping time. Take the strategy $\theta^{n+1} \triangleq \theta^{\varepsilon_{n+1}, \tau_{n}, \theta_{\tau_{n}}^{\varepsilon}}$ provided by Lemma 3.6 and put

$$
\tau_{n+1} \triangleq \inf \left\{t \geq \tau_{n}:\left|\int_{\tau_{n}}^{t} L\left(\theta_{s}, d s\right)-\int_{\tau_{n}}^{t} L\left(\theta_{s}^{n+1}, d s\right)\right|>\varepsilon_{n+1}\right\} \wedge T .
$$


This allows us to continuously extend the definition of $\theta^{\varepsilon}$ from $\left[0, \tau_{n}\right]$ to $\left[0, \tau_{n+1}\right]$ by letting $\theta^{\varepsilon} \triangleq \theta^{n+1}$ on $\left(\tau_{n}, \tau_{n+1}\right]$, and we can proceed with the next step of our inductive definition.

Since by definition of $\tau_{n+1}$ and $\theta^{n+1}$ we have

$$
\begin{aligned}
\mathbb{P}\left[\tau_{n+1}<T\right] & =\mathbb{P}\left[\sup _{\tau_{n} \leq t \leq T}\left|\int_{\tau_{n}}^{t} L\left(\theta_{s}, d s\right)-\int_{\tau_{n}}^{t} L\left(\theta_{s}^{n+1}, d s\right)\right|>\varepsilon_{n+1}\right] \\
& \leq \varepsilon_{n+1}=\varepsilon / 2^{n+1}
\end{aligned}
$$

the Borel-Cantelli Lemma implies that for $\mathbb{P}$-a.e. $\omega$ we have to carry out only a finite number of the above induction steps in order to define $\theta^{\varepsilon}(\omega,$.$) on the whole interval$ $[0, T]$. This entails, in particular, that the above procedure yields an adapted process $\theta^{\varepsilon}$ with continuous paths of bounded variation and $\theta_{T}^{\varepsilon}=\vartheta_{T}$. Moreover, we know by construction that

$$
\begin{aligned}
\sup _{0 \leq t \leq T} & \left|\int_{0}^{t} L\left(\theta_{s}, d s\right)-\int_{0}^{t} L\left(\theta_{s}^{\varepsilon}, d s\right)\right| \\
& \leq \sum_{n=0}^{+\infty} \sup _{\tau_{n} \leq t \leq \tau_{n+1}}\left|\int_{\tau_{n}}^{t} L\left(\theta_{s}, d s\right)-\int_{\tau_{n}}^{t} L\left(\theta_{s}^{n+1}, d s\right)\right|
\end{aligned}
$$

and by definition of $\tau_{n+1}$ the above sum is less than or equal to $\sum_{n=0}^{+\infty} \varepsilon_{n+1}=\varepsilon$. This completes the proof of our assertion.

Remark 3.7 Note that for the preceding theorem to hold true our continuity assumption for the paths of any primal process $P^{\vartheta}, \vartheta \in \mathbb{R}$, is essential.

As a first application of the preceding approximation results, let us characterize more explicitly which final payoffs are attainable for the large investor. This task will be carried out under the following assumption.

Assumption 4 One of the primal models, $P^{0}$ say, dominates all the others in the sense that each model $P^{\vartheta}, \vartheta \in \mathbb{R}$, admits a representation

$$
P_{t}^{\vartheta}=P_{0}^{\vartheta}+\int_{0}^{t} p_{s}^{\vartheta} d P_{s}^{0} \quad(0 \leq t \leq T)
$$

for some predictable $P^{0}$-integrable process $p^{\vartheta}$.

This assumption is clearly satisfied if the primal model $P^{0}$ is complete and Assumption 3 is satisfied. It means essentially that all primal models refer to the same exogenous shock. An important consequence of this assumption is that the set $\mathscr{P}^{*}$ of $\mathbb{P}$-equivalent probability measures under which all our primal models $P^{\vartheta}(\vartheta \in \mathbb{R})$ become local martingales coincides with the set $\mathscr{P}^{0}$ of local martingale measures for the model $P^{0}$. 
Proposition 3.8 Under Assumption 1, each density

$$
p_{s}^{\vartheta}(\omega)=\frac{d\left[P^{\vartheta}, P^{0}\right]}{d\left[P^{0}\right]}(\omega, s)
$$

occurring in Assumption 4 can be chosen continuous in $\vartheta$ for any fixed $(\omega, s) \in \Omega \times[0, T]$. The induced predictable process $p=\left(p_{s}\right)_{0 \leq s \leq T}$ with values in $C(\mathbb{R})$ allows us to write the dynamics of $L(\vartheta,)=.\int_{0}^{\vartheta} P(x,) d x,. \vartheta \in \mathbb{R}$, in the form

$$
L(\vartheta, t)=\int_{0}^{\vartheta} P(x, 0) d x+\int_{0}^{t}\left\{\int_{0}^{\vartheta} p_{s}^{x} d x\right\} d P_{s}^{0} \quad(0 \leq t \leq T) .
$$

In particular, a predictable process $\theta=\left(\theta_{t}\right)_{0 \leq t \leq T}$ is $L$-integrable provided

$$
\int_{0}^{T}\left\{\int_{0}^{\theta_{s}} p_{s}^{x} d x\right\}^{2} d\left[P^{0}\right]_{s}<+\infty \quad \mathbb{P}-\text { a.s. }
$$

and in this case we have

$$
\int_{0}^{T} L\left(\theta_{s}, d s\right)=\int_{0}^{T}\left\{\int_{0}^{\theta_{s}} p_{s}^{x} d x\right\} d P_{s}^{0} .
$$

Proof : That $p_{t}^{\vartheta}(\omega)$ can be chosen continuous in $\vartheta$ follows from Kunita (1990), Chapter 3.1. The claimed dynamics of $L(\vartheta, t)$ will follow by Fubini's theorem for stochastic integrals (cf. Protter (1990), Theorem IV.46) once we know that $\left(\left(\int_{0}^{\vartheta}\left(p_{s}^{x}\right)^{2} d x\right)^{1 / 2}\right)_{0 \leq s \leq T}$ is $P^{0}$-integrable. To prove this, note that by the standard Fubini-theorem

$$
\int_{0}^{T}\left\{\int_{0}^{\vartheta}\left(p_{s}^{x}\right)^{2} d x\right\} d\left[P^{0}\right]_{s}=\int_{0}^{\vartheta}\left\{\int_{0}^{T}\left(p_{s}^{x}\right)^{2} d\left[P^{0}\right]_{s}\right\} d x
$$

By definition of $p$, the last $\{\ldots\}$-term is almost surely equal to $\left[P^{x}\right]_{T}$. By Assumption 1 . this quantity continuously depends on $x$ and is therefore locally integrable with respect to $d x$. Hence, the last quantity in the above equation is finite almost surely.

Having established the dynamics of $L(\vartheta,$.$) it is now easy to see that \left[L(\vartheta,),. L\left(\vartheta^{\prime},.\right)\right]$ is absolutely continuous with respect to $A^{L} \triangleq\left[P^{0}\right]$ with density given by $a^{L}\left(\vartheta, \vartheta^{\prime}, s\right)=$ $\int_{0}^{\vartheta} p_{s}^{x} d x \int_{0}^{\vartheta^{\prime}} p_{s}^{y} d y$. This yields the claimed $L$-integrability criterion.

Identity (6) shows that with any large investor strategy $\theta$ we can associate a small investor strategy $\xi_{s}=\int_{0}^{\theta_{s}} p_{s}^{x} d x$ which in the model $P^{0}$ induces the same wealth dynamics as $\theta$ in our large investor model if we neglect transaction costs. We will see that the converse holds true under

Assumption 5 For $\mathbb{P} \otimes d P^{0}-$ a.e. $(\omega, s) \in \Omega \times[0, T]$, the mapping $\vartheta \mapsto \int_{0}^{\vartheta} p_{s}^{x}(\omega) d x$ is surjective from $\mathbb{R}$ onto $\mathbb{R}$. 
The preceding assumption is satisfied, if $p^{x}$ is strictly positive and does not tend to 0 'too fast' as $|x| \rightarrow \infty$, i.e., if price fluctuations are not damped 'too severely' when the large trader takes extreme positions.

Theorem 3.9 Under Assumptions 1 5, any claim $H \in L^{0}\left(\mathscr{F}_{T}\right)$ which is attainable in the small investor model $P^{0}$ is approximately attainable for the same initial capital in our large investor model (1).

Proof : Let $\xi$ be an admissible small investor strategy in the model $P^{0}$ which starting with initial capital $v$ attains some terminal payoff $H=v+\int_{0}^{T} \xi_{s} d P_{s}^{0}$. Due to Assumption 5, we can use a measurable selection result, e.g., Théorème 82 in the appendix to Chapter III of Dellacherie and Meyer (1975), to find a predictable process $\theta=\left(\theta_{s}\right)_{0 \leq s \leq T}$ such that

$$
\int_{0}^{\theta_{s}} p_{s}^{x} d x=\xi_{s} \quad \mathbb{P} \otimes d\left[P^{0}\right]_{s}-\text { a.e. }
$$

By Proposition 3.8, $P^{0}$-integrability of $\xi$ carries over to $L$-integrability of $\theta$ and

$$
\int_{0}^{T} L\left(\theta_{s}, d s\right)=\int_{0}^{T} \xi_{s} d P_{s}^{0}=H-v
$$

Hence, for the large investor, $H$ is attainable modulo transaction costs with initial capital $v$ and thus approximately attainable for the same initial capital by Theorem 3.3 .

Let us close this section with an easy consequence for the large investor's utility maximization problem:

Corollary 3.10 In the situation of Theorem 3.3, the large investor's optimal utility coincides with the maximal utility of a small investor who may invest the same initial capital in a financial market modelled by $P^{0}$. More precisely, we have

$$
\sup _{\theta \text { admissible }, V_{0-}^{\theta}=w} \mathbb{E}\left[U\left(V_{T}^{\theta}\right)\right]=\sup _{\xi \text { admissible }} \mathbb{E}\left[U\left(w+\int_{0}^{T} \xi_{s} d P_{s}^{0}\right)\right]
$$

for any concave and increasing utility function $U$, provided the right side is finite for any initial capital $w>0$

Proof : Denote by $l=l(w)$ and $r=r(w)$ the $\overline{\mathbb{R}}$-valued functions of initial capital $w>0$ defined by the left and right side of (7), respectively. As pointed out earlier, identity (6) ensures that with any large investor strategy $\theta$ we can associate a strategy $\xi$ for the small investor whose value process coincides with $w+\int_{0}^{\cdot} L\left(\theta_{s}, d s\right)$. In particular, 
$\xi$ is admissible if $\theta$ is, and we have $w+\int_{0}^{T} \xi_{s} d P_{s}^{0} \geq V_{T}^{\theta}$. This proves $l(w) \leq r(w)$. Conversely, by Theorem 3.3 , any terminal portfolio value for the small investor trading in model $P^{0}$ is approximately attainable for the large investor. Thus, we have $l(w) \geq r\left(w^{\prime}\right)$ for any $w>w^{\prime}>0$. As both $l$ and $r$ are increasing functions, the preceding arguments already show $l(w)=r(w)$ for any $w$ where $r$ does not jump. Since $r$ is also concave this is the case on the interior of its domain, i.e., on $(0,+\infty)$.

\section{Superreplication of manipulable claims}

This section discusses the pricing and hedging problem for general contingent claims whose payoff may depend on the large investor's strategy, e.g., via the price of the illiquid asset at maturity.

Let us start by introducing a convenient notion of contingent claims in our present large investor setting:

Definition 4.1 A contingent claim with maturity $T$ is specified by an $\mathscr{F}_{T} \otimes \mathscr{B}(\mathbb{R})$ measurable mapping $H$ which is bounded from below. It entitles the holder to a contingent payment of $H\left(\omega, \theta_{T}(\omega)\right)$ at time $T$ where $\theta_{T}$ denotes the large investor's position at maturity.

A prominent example for such a contingent claim is, of course, the European call option $\left(P_{T}^{\theta}-K\right)^{+}$with strike $K>0$ where $H$ can be chosen as $H(\omega, \vartheta)=\left(P_{T}^{\vartheta}(\omega)-K\right)^{+}$. Important examples not covered by the above notion are barrier options and lookback options since in both cases the complete evolution of the large investor's strategies can influence the payoff, not only his position at time $T$. It might seem more natural to define a contingent claim in an illiquid financial market as a two dimensional random variable, the two dimensions reflecting the cash and the physically settled components of a real world financial contract respectively. It is easy to see, though, that the economics of such a generalized contingent claim are equal to those of the contingent claim that is defined as the realizable portfolio value of the generalized contingent claim; see Baum (2001) for details. Finally, note that the contingent claims discussed in Section 3 are incorporated in the present setting since we can identify any random variable $H \in L^{0}\left(\mathscr{F}_{T}\right)$ with a contingent claim that does not depend on the risky asset position of the large investor.

Definition 4.2 The superreplication price of a contingent claim $H$ is the infimum of all initial capitals $v$ for which there exists an admissible strategy $\theta$ such that at time $T$ 
we have $V_{T}^{\theta} \geq H\left(\theta_{T}\right)$ almost surely:

$$
\Pi(H) \triangleq \inf \left\{v: \exists \theta \text { admissible with } V_{T}^{\theta} \geq H\left(\theta_{T}\right) \mathbb{P}-\text { a.s., } V_{0-}^{\theta}=v\right\} .
$$

Similarly, one could give a definition of superreplication prices in terms of book value instead of real value. It is not clear, however, that the liquidation proceeds from a superreplicating portfolio with respect to the book value would cover the payment obligations resulting from the claim. It therefore seems to be more appropriate to focus on superreplication with respect to realizable wealth.

The following theorem shows that essentially when the large investor seeks to determine the superreplication price for a manipulable derivative, he can first determine the terminal position $\vartheta_{T}^{*}$ in the illiquid asset which (almost) minimizes the payoff and compute then the small investor superreplication price of the induced claim $H\left(\vartheta_{T}^{*}\right)$.

Theorem 4.3 Under Assumptions 15, the superreplication price of a contingent claim $H$ satisfies

$$
\inf _{\vartheta_{T} \in L^{0}\left(\mathscr{F}_{T}\right)} \sup _{\mathbb{P}^{*} \in \mathscr{P}^{*}} \mathbb{E}^{*}\left[H\left(\vartheta_{T}\right)\right] \leq \Pi(H) \leq \inf _{\vartheta_{T} \in L^{0}\left(\mathscr{F}_{T-}\right)} \sup _{\mathbb{P}^{*} \in \mathscr{P}^{*}} \mathbb{E}^{*}\left[H\left(\vartheta_{T}\right)\right]
$$

where $\mathscr{P}^{*} \neq \varnothing$ is the set of $\mathbb{P}$-equivalent probability measures under which any primal model $P^{\vartheta}(\vartheta \in \mathbb{R})$ becomes a local martingale.

If $H$ is $\mathscr{F}_{T-} \otimes \mathbb{R}-$ measurable with $\inf _{\vartheta \in \mathbb{R}} H(\vartheta) \in L^{0}\left(\mathscr{F}_{T-}\right)$, the superreplication price is given by

$$
\Pi(H)=\sup _{\mathbb{P}^{*} \in \mathscr{P} *} \mathbb{E}^{*}\left[\inf _{\vartheta \in \mathbb{R}} H(\vartheta)\right]
$$

Proof : To prove the first ' $\leq$ '-assertion, consider an initial capital $v$ and an admissible large investor strategy $\theta$ such that $V_{0-}^{\theta}=v$ and $V_{T}^{\theta} \geq H\left(\theta_{T}\right)$ almost surely. The dynamics of $V^{\theta}$ and our monotonicity Assumption 2 entail that $V_{T}^{\theta} \leq v+\int_{0}^{T} L\left(\theta_{s}, d s\right)$. By definition, $\int_{0}^{\cdot} L\left(\theta_{s}, d s\right)$ is a local martingale under each measure $\mathbb{P}^{*} \in \mathscr{P}^{*}$ and by admissibility of $\theta$ it is bounded from below by some constant. Hence, it is a $\mathscr{P}^{*}$ supermartingale and we can conclude that

$$
v \geq v+\sup _{\mathbb{P}^{*} \in \mathscr{P}^{*}} \mathbb{E}^{*} \int_{0}^{T} L\left(\theta_{s}, d s\right) \geq \sup _{\mathbb{P}^{*} \in \mathscr{P}^{*}} \mathbb{E}^{*} V\left(\theta_{T}\right) \geq \sup _{\mathbb{P}^{*} \in \mathscr{P} *} \mathbb{E}^{*} H\left(\theta_{T}\right) .
$$

For the second ' $\leq$ '-relation, take any $\vartheta_{T} \in L^{0}\left(\mathscr{F}_{T-}\right)$ and note that, since $\mathscr{P}^{*}=\mathscr{P}^{0}$ by Assumption $4, v \triangleq \sup _{\mathbb{P}^{*} \in \mathscr{P} *} \mathbb{E}^{*} H\left(\vartheta_{T}\right)$ is the superreplication price of the claim $H\left(\vartheta_{T}\right)$ for a small investor trading in a market where the asset price follows the dynamics given by $P^{0}$; see, e.g., Kramkov (1996). Hence, there exists an admissible small investor 
strategy $\xi$ such that $H\left(\vartheta_{T}\right) \leq v+\int_{0}^{T} \xi_{s} d P_{s}^{0}$ almost surely. From Theorem 3.3 we infer that $v+\int_{0}^{T} \xi_{s} d P_{s}^{0}$ is approximately attainable for the large investor. Indeed, it follows from Theorem 3.4 that we can choose the large investor strategies $\theta^{\varepsilon}, \varepsilon>0$, whose real wealth process uniformly approximate $v+\int_{0} \xi_{s} d P_{s}^{0}$ so that at time $T$ they take the value $\theta_{T}^{\varepsilon}=\vartheta_{T}$. This ensures, that the amount $H\left(\theta_{T}\right)$ to be payed by the large investor at maturity coincides with the payoff of the 'exogenously' determined claim $H\left(\vartheta_{T}\right)$ which is superreplicated by the above strategies. Hence, the large investor can superhedge against $H$ using initial capital $v+\varepsilon$. As $\varepsilon>0$ was arbitrary, this proves $\Pi(H) \leq v$ as claimed.

Now assume that $I \triangleq \inf _{\vartheta \in \mathbb{R}} H(\vartheta)$ is $\mathscr{F}_{T-}-$ measurable and let us deduce the claimed equation for $\Pi(H)$. Let $L$ and $R$ denote the left and the right term in (8), respectively. We already know that $R \geq \Pi(H) \geq L \geq \sup _{\mathbb{P}^{*} \in \mathscr{P}^{*}} \mathbb{E}^{*}[I]$. As $I$ is $\mathscr{F}_{T-}$-measurable, we may use a measurable selection theorem, e.g., Théorème 82 in the appendix to Chapter III of Dellacherie and Meyer (1975), to find for any $\varepsilon>0$ a random variable $\vartheta_{T}^{\varepsilon} \in L^{0}\left(\mathscr{F}_{T-}\right)$ such that $\inf _{\vartheta \in \mathbb{R}} H(\vartheta) \geq H\left(\vartheta_{T}^{\varepsilon}\right)-\varepsilon$ almost surely. Hence, we have

$$
L \geq \Pi(H) \geq R \geq \sup _{\mathbb{P}^{*} \in \mathscr{P}^{*}} \mathbb{E}^{*}[I] \geq \sup _{\mathbb{P}^{*} \in \mathscr{P}^{*}} \mathbb{E}^{*}\left[H\left(\vartheta_{T}^{\varepsilon}\right)\right]-\varepsilon \geq L-\varepsilon
$$

As $\varepsilon>0$ is arbitrary, this proves $L=\Pi(H)=R=\sup _{\mathbb{P}^{*} \in \mathscr{P} *} \mathbb{E}^{*}[I]$ as claimed.

Remark 4.4 Note that the preceding argument reveals in particular that superreplication prices do not depend on the large investor's initial position $\theta_{0-}$.

A first consequence of the preceding theorem is that in the large investor framework exact replication of a contingent claim can be more expensive than superreplication, a phenomenon which also occurs in the literature on transaction costs; see Bensaid, Lense, Pages, and Scheinkman (1992), Soner, Shreve, and Cvitanić (1995) and Levental and Skorohod (1997). To see this, apply the reasoning of the above proof to a claim $H$ of the form $H=V_{T}^{\theta}$ where $\theta$ is an admissible large investor strategy with non-vanishing transaction costs $\frac{1}{2} \int_{0}^{t} P^{\prime}\left(\theta_{s-}, s\right) d[\theta]_{s}^{c}+\sum_{0 \leq s \leq t} \int_{\theta_{s-}}^{\theta_{s}}\left\{P\left(\theta_{s}, s\right)-P(x, s)\right\} d x \neq 0$.

Moreover, at first sight, the above result seems to be at odds with the PDEcharacterizations of hedging prices for large investors obtained by Frey (1998) or Schönbucher and Wilmott (2000). Note, however, that in these accounts, the trading strategies at the large investor's disposal are severely restricted: the agent is confined to use strategies which are obtained as a smooth deterministic function of the stock price. By contrast, in our setting, the large investor can use the complete information available in the market to dynamically choose his positions. In addition, Frey (1998) 
and Schönbucher and Wilmott (2000) provide hedging strategies with respect to the book value rather than the real value. As pointed out in Baum (2001), the relation

$$
W_{T}^{\theta}=V_{T}^{\theta}-L\left(\theta_{T}, T\right)+P\left(\theta_{T}, T\right) \theta_{T}
$$

between the large investor's paper value and his real value allows one to use the above techniques in order to provide analogues of our preceding results which also focus on paper value instead of real value.

\section{Conclusion}

The present paper gives a detailed analysis of general financial market models in continuous time with limited liquidity due to finite depth in the sense of Kyle (1985). It is shown that in these markets many features of the primal small investor markets carry over to the considered large investor market. In particular, absence of arbitrage for a large investor is proven to be essentially a consequence of absence of arbitrage for small investors. Moreover, attainable claims for the small investor are approximately attainable for the large investor, and similarly the computation of superreplication prices and optimal utilities carries over.

All of these results heavily rely on the assumption that trading strategies affect asset prices only via their instantaneous value. It would be desirable, though, to be able to deal also with dynamic aspects of illiquidity. Indeed, from a market microstructure perspective, every trade in the past will have a lasting effect on the trade possibilities and prices in the future, with the impact being the bigger the more recent the trade has taken place. Moreover, in the real world illiquidity can show up as bid-offer spreads or as times when only a limited amount of an asset can be traded. Finally, it would be interesting to include also the game theoretic aspects resulting from the presence of several strategically interacting large investors.

It is certainly a challenge to come up with a mathematically tractable model that covers all the features of real illiquid financial markets. Recent turmoil periods like the financial crisis in 1987, the Asian and LTCM crisis in 1998 and the boom and bust period of the internet bubble were all exacerbated if not caused by illiquidity of involved assets. Therefore, the importance of taking up this challenge can hardly be overestimated.

\section{A Appendix}

The following lemma was needed for the proof of Theorem 3.4 . 
Lemma A.1 Let $\theta$ and $\vartheta_{T}$ be as in Theorem 3.4, fix a stopping time $\tau \leq T$ and consider a random variable $\vartheta_{\tau} \in L^{0}\left(\mathscr{F}_{\tau}\right)$ with $\vartheta_{\tau}=\vartheta_{T}$ on $\{\tau=T\}$. For any $\varepsilon>0$, there exists a predictable process $\theta^{\varepsilon, \tau, \vartheta_{\tau}}$ whose paths are continuous and of bounded variation over $[\tau, T]$ such that $\theta_{\tau}^{\varepsilon, \tau, \vartheta_{\tau}}=\vartheta_{\tau}, \theta_{T}^{\varepsilon, \tau, \vartheta_{\tau}}=\vartheta_{T}$ and

$$
\mathbb{P}\left[\sup _{\tau \leq t \leq T}\left|\int_{\tau}^{t} L\left(\theta_{s}, d s\right)-\int_{\tau}^{t} L\left(\theta_{s}^{\varepsilon, \tau, \vartheta_{\tau}}, d s\right)\right| \geq \varepsilon\right] \leq \varepsilon .
$$

Proof : By construction of the stochastic integral $\int_{\tau} L\left(\theta_{s}, d s\right)$, there exists a sequence of simple processes

$$
\theta_{t}^{n}=\sum_{i=0}^{k_{n}} \theta_{i}^{n} 1_{\left(t_{i}^{n}, t_{i+1}^{n}\right]}(t) \quad(t \geq 0)
$$

with $0=t_{0}^{n}<\ldots<t_{k_{n}+1}^{n}=T$ and $\theta_{i}^{n} \in L^{0}\left(\mathscr{F}_{t_{i}^{n}}\right)$ such that $\int_{\tau}^{\cdot} L\left(\theta_{s}^{n}, d s\right)$ converges uniformly in probability to $\int_{\tau}^{\cdot} L\left(\theta_{s}, d s\right)$. In particular, there exists a simple integrand $\tilde{\theta}$ such that

$$
\mathbb{P}\left[\sup _{\tau \leq t \leq T}\left|\int_{\tau}^{t} L\left(\theta_{s}, d s\right)-\int_{\tau}^{t} L\left(\tilde{\theta}_{s}, d s\right)\right| \geq \varepsilon / 2\right] \leq \varepsilon / 2 .
$$

Now, granted our assertion holds true for simple processes such as $\tilde{\theta}$, we can find a predictable, continuous process $\theta^{\varepsilon}$ with paths of bounded variation such that $\theta_{\tau}^{\varepsilon}=\vartheta_{\tau}$, $\theta_{T}^{\varepsilon}=\vartheta_{T}$ and

$$
\mathbb{P}\left[\sup _{\tau \leq t \leq T}\left|\int_{\tau}^{t} L\left(\tilde{\theta}_{s}, d s\right)-\int_{\tau}^{t} L\left(\theta_{s}^{\varepsilon}, d s\right)\right| \geq \varepsilon / 2\right] \leq \varepsilon / 2 .
$$

Combining the preceding two probability estimates, shows that it suffices to prove the lemma for simple processes $\theta$ of the form

$$
\theta_{t}=\sum_{i=0}^{n} \theta_{i} 1_{\left(t_{i}, t_{i+1}\right]}(t) \quad(t \geq 0)
$$

with $0=t_{0}<\ldots<t_{n+1}=T$ and $\theta_{i} \in L^{0}\left(\mathscr{F}_{t_{i}}\right)$. To this end, let us introduce for $\Delta \in\left(0, \min _{i}\left\{t_{i+1}-t_{i}\right\} / 2\right)$ a piecewise linear interpolation process $\theta^{\Delta}$ as follows. On $[0, \tau)$ let $\theta^{\Delta} \triangleq 0$, and on $\left[\tau^{\Delta}, T\right]$ with $\tau^{\Delta} \triangleq \inf \{T-1 / k>\tau \vee(T-\Delta): k=1,2, \ldots\}$ put $\theta^{\Delta} \triangleq \bar{\vartheta}$ where $\bar{\vartheta}$ denotes a piecewise linear, continuous and adapted process of bounded variation with $\bar{\vartheta}_{T}=\vartheta_{T}$ which changes its slope only in $t \in\{T-1 / k: k=1,2, \ldots\}$; see Lemma A.2 below for a construction of such a process. In between $\tau$ and $\tau^{\Delta}$ define $\theta^{\Delta}$ as the continuous linear interpolation of the points in the random set of interpolation points

$$
\left\{\left(\tau, \vartheta_{\tau}\right),\left(\tau^{\Delta}, \bar{\vartheta}_{\tau^{\Delta}}\right)\right\} \cup \begin{cases}\left\{\left(t_{i}, \theta_{i-1}\right),\left(t_{i}+\Delta, \theta_{i}\right): i \leq n, \tau<t_{i}\right\} & \text { if } \tau<t_{n}, \\ \left\{\left((\tau+\Delta) \wedge(T-\Delta), \theta_{n}\right)\right\} & \text { if } t_{n} \leq \tau<T-\Delta, \\ \varnothing & \text { if } T-\Delta \leq \tau .\end{cases}
$$


By construction $\theta^{\Delta}$ is an adapted process with continuous paths of bounded variation on $[\tau, T]$ which satisfies $\theta_{\tau}^{\Delta}=\vartheta_{\tau}$ and $\theta_{T}^{\Delta}=\vartheta_{T}$. As $\Delta \downarrow 0$, we furthermore have $\theta_{t}^{\Delta}(\omega) \rightarrow \theta_{t}(\omega)$ for any $\omega \in \Omega$ and any point in time $t \in(\tau, T) \backslash\left\{t_{0}, \ldots, t_{n+1}\right\}$. Moreover, $\left|\theta_{t}^{\Delta}(\omega)\right|$ is bounded pathwise by $\max _{i}\left|\theta_{i}(\omega)\right| \vee \sup |\bar{\vartheta}(\omega)|<+\infty$. Hence, we may use dominated convergence to conclude that the quadratic variation

$$
\begin{aligned}
& {\left[\int_{\tau}^{\cdot} L\left(\theta_{s}, d s\right)-\int_{\tau}^{\cdot} L\left(\theta_{s}^{\Delta}, d s\right)\right]_{T}=} \\
& \quad \int_{\tau}^{T}\left\{a\left(\theta_{s}, \theta_{s}, s\right)-2 a\left(\theta_{s}, \theta_{s}^{\Delta}, s\right)+a\left(\theta_{s}^{\Delta}, \theta_{s}^{\Delta}, s\right)\right\} d A_{s}
\end{aligned}
$$

tends to 0 in probability as $\Delta \downarrow 0$. Here, $a\left(\vartheta, \vartheta^{\prime}, \omega, s\right)$ denotes a density of $\left[L^{\vartheta}, L^{\vartheta^{\prime}}\right](\omega)$ with respect to the process $A(\omega)$ of Assumption 1, by Kunita (1990), Chapter 3.1, this density can be chosen continuous in $\left(\vartheta, \vartheta^{\prime}\right) \in \mathbb{R} \times \mathbb{R}$ for any $(\omega, s) \in \Omega \times[0, T]$ and such that for any compact set $K \subset \mathbb{R} \times \mathbb{R},\|a(., ., \omega, s)\|_{\infty: K}$ is $d A_{s}(\omega)$-integrable for all $\omega \in \Omega$, where $\|\cdot\|_{\infty: K}$ denotes the sup-norm over $K$. It now follows from the Burkholer-DavisGundy inequality that the martingale part of $\int_{\tau} L\left(\theta_{s}^{\Delta}, d s\right)$ converges to the martingale part of $\int_{\tau} L\left(\theta_{s}, d s\right)$ uniformly in probability as $\Delta \rightarrow 0$. A similar argument also proves uniform convergence in probability of the bounded variation parts. This completes our proof.

The following lemma is needed for the construction of the process $\theta^{\Delta}$ occurring in the preceding proof.

Lemma A.2 Any random variable $\vartheta_{T} \in L^{0}\left(\mathscr{F}_{T-}\right)$ is the terminal value $\bar{\vartheta}_{T}=\vartheta_{T}$ of some continuous adapted process $\bar{\vartheta}$ with piecewise linear paths of bounded variation which change slope only in $t \in\{T-1 / k: k=1,2, \ldots\}$.

Proof : $\quad$ Let $t_{k} \triangleq(T-1 / k)^{+}(k=1,2, \ldots)$, put $f(x) \triangleq x /(1+|x|)(x \in \mathbb{R})$ and let $g \triangleq f^{-1}$ denote its inverse. By the martingale convergence theorem, the bounded martingale $M_{t_{k}} \triangleq \mathbb{E}\left[f\left(\vartheta_{T}\right) \mid \mathscr{F}_{t_{k}}\right]$ converges to $\mathbb{E}\left[f\left(\vartheta_{T}\right) \mid \mathscr{F}_{T-}\right]=f\left(\vartheta_{T}\right)$ almost surely and in $L^{1}(\mathbb{P})$. Hence, by passing to a suitable subsequence $t_{k}^{\prime}>0$, we may assume that we even have fast $L^{1}$-convergence in the sense that $\sum_{k} \mathbb{E}\left|M_{t_{k+1}^{\prime}}-M_{t_{k}^{\prime}}\right|<+\infty$.

Now, put $\bar{\vartheta}_{0} \triangleq 0, \bar{\vartheta}_{t_{k+1}^{\prime}} \triangleq g\left(M_{t_{k}^{\prime}}\right)(k=0,1, \ldots)$ (where $\left.t_{0}^{\prime} \triangleq 0\right)$, and extend the definition of $\bar{\vartheta}$ to the whole interval $[0, T]$ by letting $\bar{\vartheta}_{T} \triangleq \theta_{T}$ and linear interpolating on each interval $\left(t_{k}^{\prime}, t_{k+1}^{\prime}\right)$ between the given boundary values $\bar{\vartheta}_{t_{k}^{\prime}}=g\left(M_{t_{k-1}^{\prime}}\right), \bar{\vartheta}_{t_{k+1}^{\prime}}=g\left(M_{t_{k}^{\prime}}\right)$. This gives us an adapted process $\bar{\vartheta}$ with piecewise linear and continuous paths which 
change slope only in $\left\{t_{k}: k=1,2, \ldots\right\}$. The variation of $\bar{\vartheta}$ is given by

$$
\sum_{k=0}^{+\infty}\left|\bar{\vartheta}_{t_{k+1}^{\prime}}-\bar{\vartheta}_{t_{k}^{\prime}}\right| \leq\left|g\left(M_{0}\right)\right|+\sup _{|\vartheta| \leq \sup _{k}\left|M_{t_{k}^{\prime}}\right|}\left|g^{\prime}(\vartheta)\right| \sum_{k=1}^{+\infty}\left|M_{t_{k}^{\prime}}-M_{t_{k-1}^{\prime}}\right|
$$

As $\vartheta_{T}$ takes only finite values, $\sup _{k}\left|M_{t_{k}^{\prime}}\right|$ is strictly less than one almost surely, and therefore the above supremum over $\left|g^{\prime}(\vartheta)\right|$ yields a finite value a.s. By fast $L^{1}$-convergence of $M_{t_{k}^{\prime}}$ also the last sum is finite almost surely, and it thus follows that $\bar{\vartheta}$ has paths of bounded variation.

\section{References}

BACK, K. (1992): "Insider trading in continuous time," Review of Financial Studies, 5, 387-409.

Baum, D. (2001): "Realisierbarer Portfoliowert in illiquiden Finanzmärkten," Ph.D. thesis, Humboldt University of Berlin.

Bensaid, B., J. Lense, H. Pages, and J. Scheinkman (1992): "Derivative Asset Pricing with Transaction Costs," Mathematical Finance, 2, 63-86.

Bierbaum, J. (1997): "Über die Rückwirkung von Handelsstrategien ausgewählter Investoren auf Wertpapierpreisprozesse," Diploma Thesis, Humboldt University Berlin.

Çetin, U., R. A. Jarrow, And P. Protter (2002): "Liquidity Risk and Arbitrage Pricing Theory," Working paper, Cornell University, Ithaca.

Çetin, U., R. A. Jarrow, P. Protter, and M. Warachka (2002): "Option Pricing with Liquidity Risk," Working paper, Cornell University, Ithaca.

Cuoco, D., And J. Cvitanic (1998): "Optimal Consumption Choices for a 'Large' Investor," Journal of Economic Dynamcis and Control, 22, 401-436.

Cvitanic, J., And J. MA (1996): "Hedging Options for a Large Investor and ForwardBackward SDE's," Annals of Applied Probability, 6, 370-398.

Delbaen, F., and W. Schachermayer (1998): "The Fundamental Theorem of Asset Pricing for Unbounded Stochastic Processes," Mathematische Annalen, 312, 215-250. 
Dellacherie, C., And P. Meyer (1975): Probabilités et potentiel, Chapitres I-IV. Hermann.

Frey, R. (1998): "Perfect Option Hedging for a Large Trader," Finance and Stochastics, 2, 115-141.

Frey, R., And A. Stremme (1997): "Market Volatility and Feedback Effects from Dynamic Hedging," Mathematical Finance, 7, 351-374.

Jarrow, R. (1992): "Market Manipulation, Bubbles, Corners and Short Squeezes," Journal of Financial and Quantitative Analysis, 27, 311-336.

- (1994): "Derivative Securities Markets, Market Manipulation and Option Pricing Theory," Journal of Financial and Quantitative Analysis, 29, 241-261.

Kramkov, D. (1996): "Optional Decomposition of Supermartingales and Hedging Contingent Claims in Incomplete Security Markets," Probability Theory and Related Fields, 105, 459-479.

Kunita, H. (1990): Stochastic Flows and Stochastic Differential Equations. Cambridge University Press, Cambridge.

Kyle, A. (1985): "Continuous Auctions and Insider Trading," Econometrica, 53, 13151335.

Levental, S., And A. V. Skorohod (1997): "On the possibility of hedging options in the presence of transaction costs," Ann. Appl. Probab., 7(2), 410-443.

Papanicolaou, G., And R. Sircar (1998): "General Black-Scholes models accounting for increased market volatility from hedging strategies," Applied Mathematical Finance, 5, 45-82.

Platen, E., And M. Schweizer (1998): "On Feedback Effects from Hedging Derivatives," Mathematical Finance, 8, 67-84.

Protter, P. (1990): Stochastic Integration and Differential Equations. Springer.

Schönbucher, P. J., And P. Wilmott (2000): "The feedback effect of hedging in illiquid markets," SIAM J. Appl. Math., 61(1), 232-272 (electronic).

Soner, H. M., S. E. Shreve, and J. Cvitanić (1995): "There is no nontrivial hedging portfolio for option pricing with transaction costs," Ann. Appl. Probab., 5(2), 327-355. 
Peter BANK

Institut für Mathematik

Humboldt-Universität zu Berlin

Unter den Linden 6

D-10099 Berlin, Germany

pbank@mathematik.hu-berlin.de
Dietmar Baum

Structured Credit Products

Bank of America House

1 Alie Street

London E1 8DE, United Kingdom

dietmar. baum@bankof america.com 